\title{
Nanoparticle-Mediated Interleukin-12 Cancer Gene Therapy
}

\author{
Somayeh Hallaj-Nezhadi ${ }^{\mathrm{a}}$, Farzaneh Lotfipour ${ }^{\mathrm{a}, \mathrm{b}}$, and Crispin R. Dass ${ }^{\mathrm{c}}$ \\ ${ }^{a}$ Faculty of Pharmacy, Tabriz University of Medical Sciences, Tabriz, Iran. \\ ${ }^{\mathrm{b}}$ Biotechnology Research Center, Tabriz University of Medical Sciences, Tabriz, Iran. \\ ${ }^{c}$ School of Biomedical and Health Sciences, Victoria University, St Albans, VIC, Australia. \\ Received, May 31, 2010; Revised, October 10, 2010; Accepted, November 04, 2010; November 4, 2010.
}

\begin{abstract}
Interleukin-12 (Il-12) is a heterodimeric cytokine which has been proven to possess antitumor effects in various animal models via stimulating the immune system. However, the main problem associated with Il-12 protein delivery is its instability as well as cytotoxicity subsequent to systemic administration in rodents and in clinical trials. However, gene delivery can be used to deliver genes of interest to the tumor site. Hence, a large number of studies have been undertaken to deliver genes of interest to the tumor site through viral or non-viral vectors. Viral DNA delivery systems suffer from safety concern due to the toxicity of the viruses and strong immune response, while non-viral gene delivery systems proffer lower transfection efficiency. In contrast, nanometer-sized complexes of therapeutic DNA may prove to be more efficient for administration of therapeutic genes to solid tumors compared to administration of naked plasmid DNA. Nanoparticle-based gene delivery systems might be more pertinent, due to enhanced tissue penetrability, and improved cellular uptake. Il-12 gene delivery has already been reported with different nanoparticles containing DNA. This article provides a review on the in vivo and in vitro studies using various nanoparticles, for delivery of the Il-12 gene to neoplastic cells. The future of these promising approaches lies in the development of better techniques for preparing Il-12 gene delivery systems with complete efficiency of viral vectors in addition to the highest safety for cancer patients.
\end{abstract}

\section{INTRODUCTION}

Cancer gene therapy is the treatment of the disease by transferring DNA, RNA, small interfering RNA or antisense oligonucleotides to the site of tumor (1). In general, cancer gene therapy approaches consist of protective immune response stimulation, mutated tumor suppressor gene substitution, oncogene inactivation, suicide gene therapy, or introduction of multidrug resistance genes in bone marrow or peripheral blood stem cells (2).

It has been at least a century that the immune system has been utilized for tumor treatment, namely a treatment modality referred to as cancer immunotherapy. Cancer immunotherapy can be used to express cytokines or other immunotherapeutic agents' genes in patients in an attempt to augment the immune response to a cancer. In general, cancer immunotherapy consists of two functional methods: passive immunotherapy and active immunotherapy. Passive immunotherapy is intended to administer preformed elements of the immune system (tumor-reactive antibodies, antitumor cytokines, or tumoricidal effector cells) to patients in order to attack the cancerous cells directly. Active immunotherapy (tumor vaccines and immunostimulatory cytokines) is planned to stimulate the immune system to cause efficient antitumor immunity (3).

It has been proven that the host immune system has a main role in recognition as well as destruction of cancer cells $(4,5)$. Cytokines are regulatory proteins secreted by white blood cells and various other cells in the body. The multiple actions of cytokines include numerous effects on cells of the immune system as well as modulation of inflammatory responses (6). Cytokine genes delivery to cancerous cells as cancer gene immunotherapy alters the local tumor environment in order to induce an anti-tumor immune response to facilitate tumor eradication $(7,8)$.

Corresponding Author: Crispin R. Dass (PhD), School of Biomedical and Health Sciences, Bldg 6, Victoria University, St Albans 3021, Australia. Email: crispin.dass@vu.edu.au 
Gene transfer of cytokines or other immunotherapeutic agents for host immune response enhancement, in comparison with therapeutic protein therapy avoids the need to produce and purify large amounts of recombinant proteins. Furthermore, genetic immunotherapy has the ability to deliver immune mediators in a more efficient and safe manner. In addition, transfer of genes encoding therapeutic proteins can give rise to more "natural" sustained protein levels in vivo, and also decrease problems with immunotherapeutic agents being toxic at high doses while demonstrating short circulating half-lives (3). Usually, the expression of the cytokine was only necessary in a few cells relative to the tumor challenge, suggesting that the cytokine not only had the ability to target or kill the transfected cells alone, but was able to activate the existing antitumor immune cells in and around the tumor. Nevertheless, the induction of an inflammatory response at the site of the tumor is also possible which may result in an influx and activation of various types of cells at the tumor site (7). At the present time, there are totally $993(64.6 \%)$ clinical trials for cancer gene therapy out of totally 1537 gene therapy clinical trials. Of these, cancer gene therapy clinical trials which deliver cytokines account for $18.7 \%$ (http://www.wiley.co.uk/genetherapy/clinical/, accessed November 2009).

The general vectors used in gene delivery systems consist of viral and non-viral delivery systems. Although viral gene delivery systems possess high efficiency in various human tissues, there are concerns in relation to immunogenicity, toxicity and possible viral gene integration into the human genome. On the other hand, non-viral vectors have low toxicity and are easy to prepare. In addition, non-viral delivery systems induce no specific immune response and can carry higher amounts of genetic material without limitation on the size of the plasmid $(9,10)$. Nanoparticles (NPs) as non-viral gene delivery systems consist of polymeric NPs, liposomes, gold NPs and magnetic NPs. In fact, NPs are able to interact with and cross mucosal surfaces (11), cross blood-brain barrier, escape endo-lysosomal compartments and release genes continuously within the cell (12). In addition, NPs may give rise to enhanced cellular uptake and deeper tissue penetrability (12). According to the Ostwald-Freundlich equation, the saturation solubility was enhanced with decreasing particle size below approximately $1 \mu \mathrm{m}$. Hence NPs possess improved saturation solubility in addition to increased surface area which leads to a further increase in dissolution rate based on the NoyesWhitney equation. In contrast, the solubility of normally sized (above 1 micron) particles is a compound-specific constant which depends only on the temperature and the solvent $(13,14)$.

To date, cancer gene therapy via cytokine genes encoding interleukin-12 (Il-12) as one of the most potent anti-tumor cytokines (15), has been accomplished through viral vectors with adenovirus (16), herpes simplex virus (17), non-viral vectors including gene gun $(15,18,19)$, liposomes $(20-26)$ and electroporation (27-29). As a novelty discussion paper, this article provides a review on the recently used nanostructured delivery systems for Il-12 cancer gene therapy.

\section{INTERLEUKIN-12}

IL-12 is a heterodimeric cytokine composed of two subunits - $\alpha$-chain (35 kD as p35) and $\beta$-chain (40 $\mathrm{kD}$ as $\mathrm{p} 40$ ) covalently linked by a disulfide bridge. The two subunits of IL-12, p35 and p40, are coded by genes that are located on different chromosomes (30). The cells producing the biologically active IL12 heterodimer have the ability to produce the $\beta$ chain in free form while the free $\alpha$-chain is not secreted. While no biological function has been identified for the free $\beta$-chain, disulfide-linked homodimers of $\beta$-chain $(\mathrm{p} 40)_{2}$, in contrast to the free $\beta$-chain, can obstruct IL-12 functions in vitro and in vivo $(31,32)$.

The main stimuli that can induce the production of IL-12 include: 1) presence of microbes such as bacteria, intracellular parasites, fungi and viruses, 2) endogenous agents released by cells undergoing stress-related cell death, 3) antigen presenting cell (APC) interactions with activated $\mathrm{T}$ helper cells, and 4) interactions between APCs and extracellular matrix during inflammatory process (33). In addition, it is reported that ligation of CD4 or MHC class II molecules leads to production of IL-12 by dendritic cells (DCs), and its production is downregulated by IL-4 and IL-10 (34). Primarily produced by activated macrophages, monocytes, and dendritic cells (DCs) (35), IL-12 has a pivotal role in proinflammatory and immunoregulatory functions. The main biological activity of IL-12 is on cytotoxic $\mathrm{T}$ lymphocytes and natural killer cells 
(NK cells) which are respectively two main effectors of tumor lysis by the innate and adaptive immune responses (36). Consequently, IL-12 induces the production of interferon-gamma (IFN $\gamma$ ), tumor necrosis factor (TNF $\alpha)$, IL-2, IL-3, IL-8, IL-10, and colony-stimulating factors (CSFs) (37). Decreasing the differentiation of TH2-type helper lymphocytes and increasing the TH1-type helper immune responses (36), IL-12 has also stimulatory effects on hematopoietic precursor cells and on B lymphocytes (37).

\section{THE IMPORTANT ROLE OF INTERLEUKIN-12 IN CANCER THERAPY}

Investigations demonstrated that IL-12 possesses superior antitumor activity in comparison to other different cytokines. Furthermore, IL-12 has been shown to have efficacy in preventing inhibition of primary tumor growth induced by chemical carcinogens (37-39). IL-12 was found to improve vaccination with recombinant viruses in mice bearing micrometastases $(37,40)$. Apart from the above-mentioned comments for IL-12, this cytokine possesses potent anti-angiogenic activity produced by neutrophils, macrophages and dendritic cells (31, $41,42)$. Taken together, all these privileges explain the considerable efforts devoted to IL-12 cancer therapy. Injection of recombinant IL-12 or IL-12 gene therapy lead to the development of anti-tumor immune responses against malignant melanoma, sarcomas and mammary carcinoma in murine models (2). Since antitumor effect of IL-12 is related to the inhibition of angiogenesis (43), and the promotion of antitumor immunity, this cytokine was found to have no direct effect on tumor cells in vitro (44). Besides, exciting histological changes including a high degree of keratinization and apoptosis as well as a reduction in the proliferation rate of human tumors and extensive necrosis are found to be as other antitumor effects of IL-12 (43). To date, different tumor models have been used to investigate the anti-tumor effect of IL-12 including the B16 melanoma and its variants, C26 colon carcinoma, TSA mammary carcinoma, SCK mammary carcinoma, K1735 melanoma models, Renca renal-cell carcinoma, MC-38/colon 38 adenocarcinoma, Lewis lung carcinoma, MBT-2 bladder carcinoma, MB49 transitional-cell carcinoma, OV-HM ovarian carcinoma, M5076 reticulum-cell sarcoma, KA31 sarcoma, MCA-105 sarcoma, MCA-207 sarcoma, CSA1 M fibrosarcoma, Meth A sarcoma, X5563 lymphoma, and RAW117 lymphoma which have been reviewed in detail in literature $(31,45)$. This review focuses on cases where IL-12 gene delivery has been used for neuroblastoma therapy.

Modification of tumor cells to make them more immunogenic is a new treatment strategy. This leads to recognition and destruction of both modified and unmodified cancer cells by the immune system which results in tumor eradication (46). Tumor cells transduced in vitro with IL-12 cDNA, show induced antitumor immune responses against MCA207 murine sarcoma cell line (47). Besides, active immunization of the established P1.HTR tumor setting by IL-12 exhibited costimulation of sufficient B7 (T cell co-stimulator molecule) from the host without any need for exogenous administration (48). The strategy of using immunogenic tumor cells has been also utilized in clinical trials for immunotherapy of neuroblastoma (49) and several improved antitumour effect of IL-12, when expressed by neuroblastoma cell lines has been reported. Adenoviral-mediated IL-12 expression by tumor cells has shown promise in murine models of neuroblastoma. Cured animals showed protective immunity and cytotoxic $\mathrm{T}$ lymphocytes activity against the tumor (46).

Lode and colleagues showed that subcutaneous vaccination with a single chain IL-12 fusion protein could induce a $\mathrm{T}$ cell-mediated immunity which resulted in a complete absence of liver and bone marrow metastases in a novel poor immunogenic model of neuroblastoma (50). They also showed effective amplification of the initial $\mathrm{T}$ cell response by tumor-specific targeting of IL-2 with an antiGD2 antibody-IL-2 fusion protein (ch14.18-IL-2) after vaccination with neuroblastoma cells which are genetically engineered to produce single chain IL-12. This vaccination led to an absence of liver and bone marrow metastases after a lethal challenge with NXS2 cells only in mice vaccinated with scIL12-producing NXS2 cells and given a booster injection of low-dose ch14.18-IL-2 fusion protein (51). Siapati et al. demonstrated the improved antitumour effect of IL-2 and IL-12, when coexpressed by neuroblastoma cell lines. In vitro results support that transfection of human and mouse neuroblastoma cell lines could result in high expression levels of biologically active IL-2 and IL12. In vivo experiment showed that in a syngeneic 
neuroblastoma model, IL-2 and IL-12 expressing by transfected Neuro-2A cells could completely abolish tumorigenicity of them (52). Interesting results obtained by Croce et al. indicated that sequential vaccination with IL-12- and IL-15engineered neuroblastoma cells showed no synergistic effect but induced preventive and therapeutic effects, possibly due to the Th1 priming effect of IL-12 subsequent to the increase of CD8+ $\mathrm{T}$-cell responses and their maintenance mediated by IL-15 (53).

Cytokine-modified tumor cells using primary tumor cells from surgical biopsies are not easy to culture and have potential disadvantages preventing successful clinical application. One of the alternative strategies is to exploit fibroblasts genetically modified to secrete cytokines (54). Autologous fibroblasts in comparison with autologous tumour cells are easy to recover, manipulate in culture, and transfect using gene delivery vectors. A comparison of the antitumour effect of fibroblasts and tumour cells transfected ex vivo to coexpress IL-2 and IL-12 in a syngeneic mouse model of neuroblastoma, showed that the potency of transfected fibroblasts was equivalent to that of tumour cells. Also, syngeneic fibroblasts made therapeutic effects against established disease, and were able to generate immunological memory. Besides, coinjection of both types of cells eliminated in vivo tumorigenicity (49). A phase I dose-escalation clinical trial of peritumoral injections of IL-12-transduced autologous fibroblasts carried out in disseminated cancer patients showed promising results. Following transduction of primary dermal fibroblasts cultured from the patients with retroviral vector carrying human IL-12 genes as well as the neomycin phosphotransferase gene (TFG-hIL-12-Neo), the locoregional effects mediated by tumor necrosis factor $\alpha$ and $\mathrm{CD} 8+\mathrm{T}$ cells with tumor regression devoid of clinically significant toxicities were observed. Also, transient but clear tumor size reduction at the injected sites was shown in four of nine cases (54).

Miller and colleagues demonstrated that bone marrow-derived DCs infected with adenoviral vector expressing IL-12 are capable of activating NK cells, as well as conferring NK-mediated tumor protection in a hepatic metastasis model (55). In another investigation, increased IL-12 production by DCs expressing IL-12 showed a significant antitumor response in a poorly immunogenic murine neuroblastoma model (56). In another interesting research, IL-12 has been used as adjuvant to enhance the ability of dendritic cells DCs/tumor fusion vaccine to evoke antitumor immune responses against the liver metastasis model of C1300 neuroblastoma cells (57). DCs are potent antigen-presenting cells playing a main role in the initiation and regulation of primary immune responses. The fusion of DCs with tumor cells represents a perfect strategy to deliver, process, and present a broad array of tumor-associated antigens, including unknown tumor antigens, in the context of DCs-derived co-stimulatory molecules (58). With the transduction of IL-12 and IL-18 genes into the fusion cells (fusion/IL-12/IL-18), five times increase was observed in the level of IFN- $\gamma$. Also, a significant decrease in liver metastasis and a significant increase in survival were found in mice vaccinated with fusion/LacZ, fusion/IL-12, fusion/IL-18, or fusion/IL-12/IL-18 compared with mice given a mixture/LacZ, $\mathrm{DCs} / \mathrm{LacZ}$, or C1300/LacZ (57).

\section{THE PRIVILEGES OF INTERLEUKIN-12 GENE THERAPY VERSUS PROTEIN THERAPY}

Cancer immunotherapy via gene therapy perhaps has considerable benefits compared to related recombinant protein therapy. Administration of IL12 to animals gives rise to significant toxicity as a result of inflammatory responses produced by this cytokine $(31,59)$. In addition, severe toxicity has been reported subsequent to recombinant interleukin 12 preclinical and clinical studies (37, $60,61)$. On the other hand, delivery of protein necessitates frequent injections due to the instability of IL-12 and short half-life subsequent to bolus administration $(62,63)$. Preclinical toxicity evaluations in several animal species have documented adverse IL-12-induced hematopoietic, intestinal, hepatic, and pulmonary effects. The toxicology of IL-12 has been reviewed in detail elsewhere (64). Overall, the application of recombinant interleukin 12 to humans has been very restricted. Thus, the local administration of genes encoding IL-12 may be less toxic than systemic delivery of this cytokine as recombinant protein. 


\section{CANCER GENE THERAPY WITH NANOPARTICLES CARRYING INTERLEUKIN-12 GENE CONSTRUCTS}

Despite higher transfection efficiency of viral-based approaches, interest remains high in developing non-viral systems owing to toxicity concerns related to the viral vectors. Among the diverse non-viral based techniques, use of NPs has received increasing attention due to its unique properties such as enhanced cellular uptake, deeper tissue penetrability and escape from endolysosomal compartments.

Maheshwari et al. investigated the ability of poly [ $\alpha$-(4-aminobutyl)-L-glycolic acid] (PAGA), a biodegradable soluble nontoxic polymer as gene delivery vector (65). PAGA was developed by melting condensation and used for complex formation with plasmid encoding murine IL-12 (pmIL-12) in the presence of 5\% (w/v) glucose for murine interleukin-12 (mIL-12) gene delivery. PAGA/pmIL-12 complexes, with mean particle size of $100 \mathrm{~nm}$ were used for transfection into cultured CT-26 colon adenocarcinoma cells along with CT26 tumor-bearing $\mathrm{BALB} / \mathrm{c}$ mice via intratumoral (IT) injection. In vitro transfection demonstrated that the mIL-12 levels for PAGA/pmIL-12 complexes with $\mathrm{N} / \mathrm{P}$ mol ratio of $3: 1(3 / 1, \pm)$ were considerably higher compared to those of naked pmIL-12. Furthermore, at $48 \mathrm{~h}$ after intratumoral injection of polylexes, tumors were harvested, chopped, and recultured for $24 \mathrm{~h}$ and the culture supernatants were studied by ELISA. According to the results, higher levels of mIL-12 was produced following injection of PAGA/pmIL-12 complexes $(3 / 1, \pm)$ in comparison with naked pmIL-12. Also due to IL-12 capability of mIFN- $\gamma$ induction, the level of this cytokine was studied. Based on the results PAGA/pmIL-12 complexes produced much higher levels of mIFN- $\gamma$ compared to naked pmIL12. Besides, the persistence of mIL-12 expression was investigated by RT-PCR at 1,3 , and 7 days after IT injection of PAGA/pmIL-12 complexes $(3 / 1, \pm)$ to CT-26 tumor-bearing BALB/c mice. The level of mIL-12 p35 expression on day 1 and day 3 showed significantly higher levels of mIL-12 compared to days 3,5 , and is nearly negligible on day 7. In addition, single-dose IT administration of the polyplex over a period of 56 days showed retardation of tumor progression with life span compared to mice injected with pmIL-12 and 5\% glucose alone. Additionally, dimethylthiazolyl- diphenyltetrazolium bromide (MTT) assay of PAGA/pmIL-12 complexes for testing cytotoxicity in CT-26 cells revealed that polyplexes were nontoxic to the cells when formulated at a charge ratio of $7 / 1( \pm)$ and below. Taken together, the authors envisaged that PAGA as nontoxic and biodegradable polymer can be used for repeated injections to maintain sustained gene expression for cancer treatment.

In one study reported by Mahato et al., a watersoluble lipopolymer (WSLP) was synthesized for delivery of the IL-12 gene (66). WSLP/p2CMVmIL-12 complexes, with mean particle size of 26 to $62 \mathrm{~nm}$, were prepared using branched polyethylenimine (PEI) of $1800 \mathrm{Da}$ and cholesteryl chloroformate, and constructed p2CMVmIL-12, encoding the IL-12 subunits p35 and $\mathrm{p} 40$, each under the transcriptional control of a separate cytomegalovirus (CMV) promoter. Transfection efficiency of complexes of branched PEIs of different molecular weights $(1800,10,000$ and 25,000 Da) with p2CMVmIL-12 was studied in vitro in CT-26 cells. Transfection efficiency of formulations with PEI of 1800 was found to be very low but increased with increasing molecular weight of PEI. The formulations prepared using 25,000 Da PEI, was very toxic to the cells, while demonstrating the highest transfection efficiency among all the PEI-based formulations. Interestingly, WSLP/p2CMVmIL-12 complexes showed higher transfection in CT-26 cells in comparison with the PEI based formulations. With an increase in N/P ratios from 5 to 15 , transfection efficiency of the WSLP/p2CMVmIL-12 complexes enhanced, but then there was no significant increase in transfection up to the N/P ratio of 30/1. In addition, the effect of plasmid construct design on IL-12 expression in CT-26 cells was studied comparing the complexes prepared with p2CMVmIL-12 and pIRESmIL-12. Both plasmids encoded for IL-12; however, in p2CMVmIL-12, each of the p35 and p40 subunits were encoded under the transcriptional control of a separate cytomegalovirus (CMV) promoter, while in pIRESmIL-12 the p35 and p40 subunits were linked to the internal ribosome entry sites (IRES). The results revealed that free or complexed p2CMVmIL-12 had higher IL-12 expression compared with free or complexed pIRESmIL-12. Besides, the gene expression levels of IL-12 and induced IFN- $\gamma$ after IT injection of WSLP/p2CMVmIL-12 complexes into BALB/c 
mice bearing CT-26 subcutaneous tumors was investigated. 48 hours subsequent to IT injection, tumors were harvested, chopped, and recultured for 24 hours, and then culture supernatants were analyzed by ELISA. Based on the results, the levels of IL-12, IFN- $\gamma$, and nitric oxide (which its production is regulated by IL-12) was found to be higher than those of naked p2CMVmIL-12, WSLP, and $5 \%$ glucose. In addition, to study the persistence of the gene expression after IT injection of WSLP/p2CMVmIL-12 complex, tumors were isolated, chopped, and cultured for IL-12 at days 1, 3 , and 5 postinjection. At day 1, IL-12 levels were highest decreasing slowly with time. At day 3, IL12 levels were higher for WSLP/p2CMVmIL-12 complex than those for the naked DNA. Furthermore, survival rate and tumor growth inhibition was significantly improved following a single injection of WSLP/p2CMVmIL-12 complexes.

In another experiment, the same research group synthesized WSLP/ p2CMVmIL-12 complexes using branched PEI of $1800 \mathrm{Da}$ and cholesteryl chloroformate at N/P mol ratio of $20: 1$ in the presence of $5 \%(\mathrm{w} / \mathrm{v})$ glucose $(62)$. The resultant WSLP/ p2CMVmIL-12 complexes with mean diameter of $61.8 \mathrm{~nm}$, was examined to study the organ distribution and anti-tumor response in vivo in CT-26 subcutaneous tumor-bearing BALB/c mice after repeated IT injection at 4- and 8- day intervals. The most significant anti-tumor activity was shown in mice receiving treatment at an interval of 4 and 8 days compared to those received a single dose. The mean tumor volume at 30 days for mice receiving repeat injections at 4- and 8-day intervals was less than $3000 \mathrm{~m}$, while it was greater than 5000 for those receiving a single dose. Also PEI 25K/p2CMVmIL-12 complexes as well as naked p2CMVmIL-12 formulated in $5 \%(\mathrm{w} / \mathrm{v})$ glucose was investigated as controls. Lower antitumor activity was reported in mice injected with PEI 25K/p2CMVmIL-12 compared to WSLP/p2CMVmIL-12 formulations. Furthermore, relatively similar or more potent anti-tumor activity was observed for mice treated with naked p2CMVmIL-12. Organ distribution subsequent to IT injection of WSLP/ p2CMVmIL-12 complexes to CT-26 subcutaneous tumor-bearing $\mathrm{BALB} / \mathrm{c}$ mice was studied using different dyes with different emission spectrum and minimal overlap to label WSLP and p2CMVmIL-12 separately. Based on the results, enhanced retention of either labeled WSLP or p2CMVmIL-12 within the tumor for $24 \mathrm{~h}$ and limited accumulation in other organs (lung, heart, kidney, liver, spleen and blood) for a period of $96 \mathrm{~h}$ was observed.

In 2005, Fewell et al. investigated the ability of synthetic polymeric delivery vehicle (PPC) for interleukin-12 gene (pmIL-12) delivery (67). PPC was synthesized by covalently conjugating a low molecular weight branched polyethylenimine (PEI) with cholesterol (CHOL) and methoxypolyethyleneglycol (PEG). PPC as a gene delivery polymer was able to form stable nanocomplexes with DNA as well as protect DNA from degradation by DNase. The ratio of PEG/PEI/CHOL was optimized for physicochemical properties and gene delivery efficiency of PPC polyplexes encoding for luciferase following intratumoral injection into 4T-1 murine mammary tumors in vivo. Also In vitro optimization of the ratio of nitrogen to phosphate $(\mathrm{N} / \mathrm{P})$ between PPC polymer and DNA was done in COS-1 cells. Dependent on the amount of DNA used, in vitro administration of pmIL-12/PPC polyplexes (N/P ratio of 11:1) showed high levels of mIL-12 production in COS-1 cells. Following administration of pmIL-12/PPC polyplexes in mice bearing an established subcutaneous 4T-1 tumor model led to significant inhibition of tumor growth in combination with paclitaxel as a chemotherapeutic agents (Genexol-PM; 2 treatments, every other week) and Plasmid administrations (3 weekly injections) alone. In addition, almost complete tumor inhibition was observed when the treatments were combined. Furthermore, pmIL-12/PPC administration against the mouse bearing a murine squamous-cell carcinoma model (SCCVII) in combination with cyclophosphamide (Cytoxank; given either 1 day or 5 days prior to plasmid administration) revealed a significant reduction in tumor growth compared to pmIL-12/PPC treatment alone. The group treated with Cytoxan 5 days prior to treatment with pmIL12/PPC demonstrated significant tumor inhibition in comparison with was the Cytoxan treatment alone group; whereas the group received Cytoxan 1 day prior to $\mathrm{mIL} 12 / \mathrm{PPC}$ revealed slight difference from the Cytoxan alone group. As a whole, the authors concluded that pIL-12/PPC probably have a potential utility as an adjuvant therapy for cancer treatment. 
In another investigation, Fewell et al. studied PPC via IP administration into a mouse model of disseminated ovarian cancer (68). IP administration of pmIL-12/PPC led to elevated protein levels of both murine IL-12 (mIL-12) and interferon (IFN)- $\gamma$ $(\mathrm{mIFN}-\gamma)$ in ascites fluid, with little change observed systemically. The mIL-12 protein was detectable for several days and could be reintroduced by weekly injections. Moreover, ovarian cancer is described by the rapid growth of solid IP tumors and massive ascites. Vascular endothelial growth factor (VEGF) enhances tumor growth by inducing neovascularization and may develop ascites formation by vascular leakage. $(69,70)$. Fewell and coworkers reported that following pmIL-12/PPC IP administration in tumor model, expression of IL-12 led to a significant decrease in VEGF protein which resulted in inhibition of ascites accumulation. Also, there was not significant evidence of systemic toxicity following a comprehensive safety/toxicity analysis of four weekly i.p. injections of pmIL-12/PPC in healthy animals. Overall, pmIL-12/PPC via IP route may give rise to limitation of gene expression in sites where malignancies are located as well as reduction of systemic toxicities (68).

Wang et al. prepared a biodegradable cationic amphiphilic copolymer composed of a cationic main chain, (poly N-methyldietheneamine sebacate, (PMDS)), and cholesterol side chains (71). Cationic core-shell NPs were prepared self-assembly form the copolymer, poly $\{(\mathrm{N}$-methyldietheneamine sebacate)-co-[(cholesteryl oxocarbonylamido ethyl) methyl bis(ethylene) ammonium bromide] sebacate\}, P(MDS-co-CES). Paclitaxel, indomethacin and pyrene were encapsulated as model drugs first in the core-shell NPs to investigate the possibility of the copolymer to codeliver a drug and DNA. The polymer particle size increased from $83 \pm 1$ to $119 \pm 3,175 \pm 2$, and $180 \pm$ $2 \mathrm{~nm}$ after loading with paclitaxel, indometbacin and pyrene, correspondingly. The zeta potential of the NPs decreased from $84 \pm 5$ to $68 \pm 3,63 \pm 2$ and $65 \pm 1 \mathrm{mV}$, respectively. Then plasmid DNA encoding the $6.4 \mathrm{~kb}$ firefly luciferase (pCMVluciferase VR1255_C) bound to the blank core-shell NPs and paclitaxel, indometbacin and pyreneloaded core-shell NPs. Owing to the lower zeta potential of the drug-loaded core-shell NPs, DNAbinding ability of them was a little lower than that of the blank core-shell NPs. The structural integrity of the drug-loaded core-shell NPs during the DNA binding process was studied using the microenvironment of the pyrene-loaded NPs with luminescene spectrometer. The findings indicated that pyrene-loaded NPs did not collapse during DNA binding proving the ability of the core-shell NPs to carry a drug and DNA simultaneously in a stable colloid. Subsequently IL-12-encoded plasmid DNA was bound to the cationic shell of the paclitaxel-loaded NPs. The vivo transfection experiments were carried out on female Balb/c mice bearing subcutaneous 4T1 breast tumours which share many characteristics with human breast tumors. Paclitaxel-loaded NPs/ IL-12-encoded plasmid complexes led to a significant inhibition of tumor growth in combination with mice treated with either paclitaxel-loaded NPs or NPs 1L-12 gene complexes $(\mathrm{P}<(0.001)$, indicating a significant synergistic combined effect of co-delivery of the drug and the IL-12 gene.

Kim et al. presented mannosylated chitosan (MC) NPs to induce mannose receptor-mediated endocytosis of IL-12 gene directly into dendritic cells which reside within the tumor (72). The MC/ DNA complex at charge ratios of 5 to 20 were found to be stable enough to carry the DNA and pmIL-12 was effectively protected from degradation by DNase I. The atomic force microscopy image of MC/DNA polyplex (N/P: 5) indicated spherical or ellipsoidal structure with average particle sizes of 136.7 F $33.5 \mathrm{~nm}$. Moreover, MC exhibited less toxicity than chitosan in NCTC 3749 macrophage cells over a wide range of concentrations. To determine whether gene delivery with MC is mannose receptor-mediated, initial in vitro studies carried out on Raw 264.7 macrophage cells expressing moderate mannose receptors. It was found that MC-mediated pGL3 DNA transfection was mannose receptor-dependent since mannose could block the binding in a concentration-dependent manner. Subsequently in vitro studies in dendritic cells collected from mice demonstrated that the level of mIL-12 p70 was increased by MC/pmIL-12 compared with chitosan/pmIL-12 complexes and naked mIL-12. In vivo studies showed that after IT injection of $\mathrm{MC} /$ mIL-12 complex into BALB/c mice bearing CT-26 carcinoma cells at a dose of $50 \mu \mathrm{g}$ of DNA per mouse, at 3-day intervals for a total of four treatments (on days $0,3,6$,and 9) inhibited tumor growth and angiogenesis, and significantly induced 
cell cycle arrest and apoptosis. Moreover, MC/pmIL-12 complex facilitated apoptosis in tumor tissues. In general, anticancer drugs could lead to an apoptosis as a result of balanced transcription of antiapoptotic genes such as Bcl-xL and proapoptotic genes such as Bad and Bax. Based on the Western blotting analysis, the expression levels of proapoptotic Bad, Bax, and Apaf-1 in treated groups were highly increased compared to control groups whereas that of antiapoptotic Bcl-xL reduced significantly after $\mathrm{MC} / \mathrm{pmIL}-12$ gene delivery. Also, administration of $\mathrm{MC} / \mathrm{pmIL}-12$ complex demonstrated antiangiogenesis by losing the activities of matrix metalloproteinase-2, vascular endothelial growth factor (VEGF), and fibroblast growth factor-2 (FGF-2) as the beginning steps of tumor angiogenesis. Thus, mannosylated chitosan (MC) NPs may be helpful as a new cytokine gene delivery system for cancer immunegene-therapy.

$\mathrm{D}^{\prime} 1 \mathrm{ez}$ et al in 2009 developed Asialoglycoprotein receptor-targeted cationic NPs for IL-12 encapsulation (NP1) or adsorption (NP2) by blending poly(D,L-lactic-co-glycolic) acid (PLGA) (50:50) with the cationic lipid 1,2dioleoyl-3-(trimethylammonium) propane (DOTAP) and the ligand asialofetuin (AF) (73). Asialofetuin (AF), a glycoprotein ligand with triantennary galactose terminal sugar chains can bind to the asialoglycoprotein receptor (ASGPr), which is specifically and abundantly expressed on the surface of normal hepatocytes and overexpressed in hepatocarcinoma cells. Cationic NPs were prepared via a modified solvent evaporation process. In the NP1 particles, the plasmid DNA was encapsulated into the cationic PLGA DOTAP NPs, while in the NP2 particles, the plasmid DNA was adsorbed on the cationic surface of a mixture of anionic PLGA particles and cationic DOTAP liposomes. NPs sizes were found to be in the range $351 \pm 40$ to $426 \pm 26 \mathrm{~nm}$, with unimodal size distribution and there were no significant differences in the size of particles prepared with encapsulated (NP1) or adsorbed DNA (NP2) in the presence of pCMVLuc. The anti-tumor effect of the prepared NPs was examined in vivo in BNL (undifferentiated murine hepatocarcinoma) subcutaneous tumor-bearing $\mathrm{BALB} / \mathrm{c}$ mice after single IT injection. Mice injected with plain-NP1 or AF-NP1 showed significant BNL-derived tumor growth inhibition $(\mathrm{p}<0.01)$. Also, $75 \%(6 / 8)$ and
$37.5 \%(3 / 8)$ of mice treated with AFNP1 and plainNP1 correspondingly, demonstrated complete tumor regressions without signs of recurrence. Furthermore, animal survival improved noticeably by injection of AF-NP1 in the presence of the therapeutic gene, with a survival rate of $75 \%$ and $37.5 \%$ in mice injected with AF- and plain-NP1, correspondingly. High levels of IL-12 and interferon- $\gamma$ were detected in the sera of Balb-c mice bearing BNL tumors after injection of the formulations. Hence, a targeted non-viral vector for IL-12 gene transfer in hepatocellular carcinoma, via non-toxic cationic PLGA/DOTAP/AF NPs was developed.

In another investigation, the same research group examined the AF-NPs carrying genes encoding for 1L-12 and luciferase in vitro in human hepatocellular liver carcinoma cell line (HepG2) cells in the absence or presence of serum (FBS) (74). Plain and AF-NPs were investigated in the presence of pCMVIL-12 to examine the effect of AF on the transfection activity in HepG2 cells. NPs containing the ligand demonstrated higher values of expression compared to the plain (non-targeted) ones. The IL-12 expression in the presence of asialofetuin increased 2- fold for both NP1 and NP2 particles. A higher value of gene expression was observed in the case of NP1 formulations compared to NP2 particles. Also, the viability of the transfected cells $(80 \%)$ ) was not changed in the presence of the ligand. In addition, the loading efficiencies of the PLGA-DOTAP NPI and NP2 were found to be respectively $95 \pm 5 \%$ and $99 \pm$ $4 \%$. Also, the formulations possessed a surface positive charge owing to the inclusion of the cationic lipid DOTAP in the preparation of the NPs; the zeta potential of the NP2 particles was smaller than that of NPl $(14 \pm 2$ vs. $37 \pm 2 \mathrm{mV}$ before lyophilization). Moreover, the PLGA-DOTAP NPs protected the plasmid against degradation over a 30min of incubation with the enzyme time period whereas naked DNA was degraded in the first 5 min. Furthermore, comparative cellular uptakes were studied using two different cell lines, HepG2 $(\mathrm{ASGPr}+)$ and HeLa (ASGPr-, defective in asialoglycoprotein receptors), to determine the intracellular AF receptor-mediated specific delivery of AF-NP. Based on the results, in HepG2 cells there was an increase in gene expression, while no increase in transfection efficiency of HeLa cells was observed, confirming the targeted intracellular 
delivery of AF-NP via an AF-receptor-mediated endocytosis mechanism. Since AF-particles can interact with the cell surface receptors, resulting in the association of asialofetuin which facilitate the internalization of the complex owing to the ability of the ligand to stimulate endocytosis. Thus, asialoglycoprotein receptor targeting by PLGADOTAP-DNA NPs carrying IL-12 can be used in gene delivery to liver cancer cells.

Rodrigo-Garzo' $n$ and coworkers

investigated the antitumoral efficacy of the systemic administration of PEI-DNA NPs carrying a plasmid codifying for IL12 (PEI-IL12) for the treatment of lung cancer and pulmonary metastases in a murine model. Single dose of PEI-DNA NPs carrying plasmids containing the IL12, or $\beta$-galactosidase genes under the control of the human cytomegalovirus promoter were injected intravenously to C57BL/6J mice with Lung cancer induced by tail vein injection of LLC cells and $\mathrm{BALB} / \mathrm{c}$ with pulmonary metastases induced by tail vein injection of CT26 cells in BALB/c mice. In both models, survival rates and immune response were evaluated. Based on the results, differences in the response to the treatment depended on the animal model. For instance, in a lung cancer model, the survival was similar between PEI-LacZ and PEI-IL12 complexes, and immunological memory was not generated, since all mice that survived the first injection died after a second LLC tumor cell injection, that is they were not protected on rechallenge. Conversely, in the model of pulmonary metastasis with CT26, the survival rate was noticeably higher in mice treated with PEI-IL12 than in mice received PEI-LacZ. Besides, in the model of pulmonary metastasis with CT26, immunological memory was generated, since the survived mice rejected a second tumor cell injection. Moreover, when the pulmonary metastases were treated, only the administration of PEI-IL12 protects against tumor development. Hence, the characteristics of the immune response after PEI-DNA injection were found to be different between both models. As the immune response that was generated in the model of lung cancer was found to be non-specific by ELISPOT analysis, and the survived mice were not protected on rechallenge; whereas in the model of pulmonary metastasis of colon cancer, specific cytotoxic $\mathrm{T}$ lymphocytes and an immunological memory were generated. Also, there were significant differences between the number of IFN- $\gamma$-producing cells in the groups treated with PEI-DNA NPs and the untreated group.

Furthermore, as mentioned above, most of the animals rejected a second injection of tumor cells. The chief differences between both models are the tumor cell line and the strain of mice. Indeed, CT26 is highly immunogenic while LLC is a poorly immunogenic tumor cell line. More experiments should be done on different tumor cell lines in the same strain of mice in order to shed light on reasons for this phenomenon. In addition, in the case of lung cancer model, the analysis of cytokine explained the issue for the strong antitumoral efficacy shown in mice treated with PEI-LacZ. Similar levels of IL12 and IFN- $\gamma$ were reached in the serum of the mice receiving PEI-LacZ and PEI-IL12 complexes. Nevertheless, the duration of IL12 expression was longer in the mice receiving PEI-IL12 complexes. Activation of macrophages or dendritric cells transduced by PEI-DNA complexes got activated by the non-methylated $\mathrm{CpG}$ sequence present in both plasmids and produce elevated amounts of IL12 which in turn could induce IFN- $\gamma$ secretion by NK and NKT cells and enhance their cytolitic activity. Non-methylated $\mathrm{CpG}$ sequences are frequently found in DNA of bacterial origin which recognized as pathogen-associated molecular patterns by the immune system of the mammals. Thus, in the case of lung cancer model, the antitumoral activity is due to the activation of the innate immune system by the $\mathrm{CpG}$ motifs.

On the other hand, in CT26 model, the antitumoral activity of local recombinant IL12 expression has a major roll. Since significant differences were found between PEI-LacZ and PEI-IL12 groups, as in PEI-IL12 group five out of six mice express IL12 out to day 5, whereas in PEILacZ group, IL12 was detected only in two out of six mice. This difference in the duration of IL12 expression may explain the higher antitumoral efficacy found in the PEI-IL12 group in the CT26 models. Taken together, the intravenous administration of PEI-DNA NPs can inhibit lung cancer and pulmonary metastases development via strong innate immune response.

\section{FUTURE DIRECTIONS}

In general, non-viral delivery systems compared to the viral vectors, induce no specific immune response and can carry higher amounts of genetic 
material with no limitation on the size of the plasmid. The ideal non-viral gene vector for cancer therapy is supposed to be low toxic, biocompatible and biodegradable, easily prepared and scaled-up at low cost and stable in storage. It should also be capable of particular gene expression in target cells and have little, if any, interaction with plasma proteins. Favorably, NPs are able to interact with and cross mucosal surfaces, cross blood-brain barrier, escape endo-lysosomal compartments and release genes continuously within the cell. In addition, NPs may induce enhanced cellular uptake and deeper tissue penetrability. In view of the fact that present cancer gene therapy using NPs is lacking in both efficiency and specificity in comparison with viral vectors, thus, much work remains to be done in the future. Furthermore, since in the case of disseminated and deep-seated tumors, systemic application is considered as a required administration route particularly (76), better methods to prepare non toxic receptor- targeted NPs are needed for improved systemic gene therapy for IL-12 gene delivery. Generally, for systemic gene delivery, IL-12 gene must be protected from degradation in the biological environment, extravasate and diffuse throughout the tissues to achieve the target site as well as have an adequate long circulation time in the blood. In addition, being inert against non-specific interactions with biological fluid, extracellular matrix and non-target cells are required. In contrast, the genetic material should have the ability of specific uptake into the target cells (77). These will necessitate the improvement of further effective and patientcompliant techniques to increase the potency of NPbased IL-12 gene delivery systems compared to that of viral vectors.

The preferred non-viral gene vector for preparation of NPs carrying a plasmid coding for IL12 in cancer therapy can be managed by optimization of existing gene delivery systems which are considered to be more efficient and can be safely used for gene therapy with no toxicity and immunogenicity concerns. The other possibility is to improve the chemical structure of the existing polymers to synthesize more cationic vectors, leading to more efficient interactions with anionic nucleic acid sequences. Another option is to engineer modified polymers so as to target the gene to the cancerous cells and ensure that the plasmid coding for IL12 is just expressed at the target cell, for instance, by use of ligand complexes. The other possibility is combination of the plasmid coding for IL12 with conventional cancer therapy like chemotherapy or radiotherapy. Finally, enough experiments in various human cancerous cells, as well as in animals are needed to assess the efficacy and safety of NPs carrying a plasmid coding for IL12 in cancer therapy.

\section{SUMMARY}

Satisfactorily, IL-12 possesses superior antitumor as well as potent anti-angiogenic activities in comparison with the different cytokines. All these privileges have led to the considerable efforts devoted to the IL-12 cancer therapy. Instability of Il-12 protein as well as its cytotoxicity is considered to be the main problem associated with Il-12 protein delivery following systemic administration. Nonetheless, gene delivery can be used to deliver desired genes to the tumor site. Nanoparticle-based gene delivery systems might be more pertinent, since nanometer-size complex of therapeutic DNA perhaps be more efficient for administration of therapeutic genes to solid tumors compared to administration of naked plasmid DNA. In this article, we have reviewed the investigations that have used NP-based IL-12 gene delivery systems in vivo and in vitro, and have related them to cancer gene therapy. A summary table (Table 1) is provided to list the major studies in this area. 
Table 1. Major studies looking at nanoparticulate delivery of IL-12 gene vectors.

\begin{tabular}{|c|c|c|c|c|c|}
\hline Gene type & Vector & Cancer type & $\begin{array}{l}\text { In vitro/in } \\
\text { vivo }\end{array}$ & & Ref. \\
\hline mIL-12 & $\begin{array}{l}\text { poly[ } \alpha \text {-(4-aminobutyl)-L- } \\
\text { glycolic acid] (PAGA) }\end{array}$ & CT-26 cells & $\begin{array}{l}\text { in vitro and in } \\
\text { vivo }\end{array}$ & Intratumoral & $(65)$ \\
\hline p2CMVmIL-12 & $\begin{array}{l}\text { water-soluble lipopolymer } \\
\text { (WSLP) }\end{array}$ & CT-26 cells & $\begin{array}{l}\text { in vitro and in } \\
\text { vivo }\end{array}$ & Intratumoral & (66) \\
\hline p2CMVmIL-12 & $\begin{array}{l}\text { Water soluble lipopolymers } \\
\text { using cholesteryl chloroformate } \\
\text { and PEI }\end{array}$ & CT-26 cells & in vivo & Intratumoral & $(62)$ \\
\hline mIL-12 & $\begin{array}{l}\text { polyethylenimine covalently } \\
\text { linked to cholesterol } \\
\text { (CHOL) and } \\
\text { methoxypolyethyleneglycol } \\
\text { (PEG) molecules forming } \\
\text { PEG-PEI-CHOL (PPC). }\end{array}$ & $\begin{array}{l}\text { 4T-1 cell lines were } \\
\text { established from a } \\
\text { spontaneous, } \\
\text { moderately } \\
\text { differentiated } \\
\text { adenocarcinoma } \\
\text { growing in Balb/C mice }\end{array}$ & $\begin{array}{l}\text { in vitro and in } \\
\text { vivo }\end{array}$ & Intratumoral & (67) \\
\hline IL-12 plasmid & $\begin{array}{l}\text { puly(N-lnethyldietheneamine } \\
\text { sebacate) (PMDS) and } \\
\text { cholesterol }\end{array}$ & $\begin{array}{c}4 \mathrm{~T} 1 \text { mouse } \\
\text { breast cancer }\end{array}$ & $\begin{array}{l}\text { in vitro and in } \\
\text { vivo }\end{array}$ & Intratumoral & (71) \\
\hline pmIL-12 & Mannosylated chitosan & CT-26 cells & $\begin{array}{l}\text { in vitro and in } \\
\text { vivo }\end{array}$ & Intratumoral & (72) \\
\hline pmIL-12 & $\begin{array}{l}\text { covalently conjugated } \\
\text { polyethylenimine } \\
\text { with cholesteryl Chloroformate } \\
\text { and } \\
\text { methoxypolyethyleneglycol }\end{array}$ & ovarian cancer & $\begin{array}{l}\text { in vitro and in } \\
\text { vivo }\end{array}$ & Intratumoral & $(68)$ \\
\hline pCMV IL-12 & $\begin{array}{l}\text { poly(D,L-lactic-co-glycolic) } \\
\text { acid (PLGA) }(50: 50) \text { with } \\
\text { the cationic lipid } 1,2 \text {-dioleoyl- } \\
\text { 3-(trimethylammonium) } \\
\text { propane (DOTAP) and the } \\
\text { ligand asialofetuin (AF) }\end{array}$ & $\begin{array}{l}\text { BNL (undifferentiated } \\
\text { murine } \\
\text { hepatocarcinoma) cells }\end{array}$ & in vivo & Intratumoral & (73) \\
\hline IL-12 plasmid & Polyethylenimine & lung cancer treatment & in vivo & intravenous & (75) \\
\hline
\end{tabular}

\section{REFERENCES}

1. Weir B, Zhao X, Meyerson M. Somatic alterations in the human cancer genome. Cancer Cell. 2004 Nov;6(5):433-8.

2. Habib NA. Cancer Gene Therapy Past Achievements and Future Challenges. New York: Kluwer Academic Publishers; 2000.

3. David T. Curiel, Douglas JT. Cancer Gene Therapy. Totowa, NJ: Humana Press Inc.; 2005

4. Vitolo D, Letessier EM, Johnson JT, Whiteside TL. Immunologic effector cells in head and neck cancer. J Natl Cancer Inst Monogr. 1992(13):203-8.
5. Cortesina G, De Stefani A, Sacchi M, Rosso S, Galeazzi E. Immunomodulation therapy for squamous cell carcinoma of the head and neck. Head Neck. 1993 May-Jun;15(3):266-70.

6. Thomson AW, Lotze MT. The Cytokine Handbook. 4th ed. London: Academic Press; 2003.

7. Kresina TF. An Introduction to Molecular Medicine and Gene Therapy. New York: A JOHN WILEY \& SONS, INC.; 2001.

8. Brunda MJ, Luistro L, Warrier RR, Wright RB, Hubbard BR, Murphy M, et al. Antitumor and antimetastatic activity of interleukin 12 against 
murine tumors. J Exp Med. 1993 Oct 1;178(4):122330.

9. Davis ME. Non-viral gene delivery systems. Curr Opin Biotechnol. 2002 Apr;13(2):128-31.

10. Schmidt-Wolf GD, Schmidt-Wolf IG. Non-viral and hybrid vectors in human gene therapy: an update. Trends Mol Med. 2003 Feb;9(2):67-72.

11. Alonso MJ. Nanomedicines for overcoming biological barriers. Biomed Pharmacother. 2004 Apr;58(3):168-72.

12. Basarkar A, Singh J. Nanoparticulate systems for polynucleotide delivery. Int $\mathrm{J}$ Nanomedicine. 2007;2(3):353-60.

13. Muller RH, Keck CM. Challenges and solutions for the delivery of biotech drugs--a review of drug nanocrystal technology and lipid nanoparticles. J Biotechnol. 2004 Sep 30;113(1-3):151-70.

14. Muller RH, Jacobs C, Kayser O. Nanosuspensions as particulate drug formulations in therapy. Rationale for development and what we can expect for the future. Adv Drug Deliv Rev. 2001 Mar 23;47(1):3-19.

15. Rakhmilevich AL, Janssen K, Turner J, Culp J, Yang NS. Cytokine gene therapy of cancer using gene gun technology: superior antitumor activity of interleukin-12. Hum Gene Ther. 1997 Jul 20;8(11):1303-11.

16. Emtage PC, Wan Y, Hitt M, Graham FL, Muller WJ, Zlotnik A, et al. Adenoviral vectors expressing lymphotactin and interleukin 2 or lymphotactin and interleukin 12 synergize to facilitate tumor regression in murine breast cancer models. Hum Gene Ther. 1999 Mar 20;10(5):697-709.

17. Parker JN, Gillespie GY, Love CE, Randall S, Whitley RJ, Markert JM. Engineered herpes simplex virus expressing IL-12 in the treatment of experimental murine brain tumors. Proc Natl Acad Sci U S A. 2000 Feb 29;97(5):2208-13.

18. Rakhmilevich AL, Turner J, Ford MJ, McCabe D, Sun WH, Sondel PM, et al. Gene gun-mediated skin transfection with interleukin 12 gene results in regression of established primary and metastatic murine tumors. Proc Natl Acad Sci U S A. 1996 Jun 25;93(13):6291-6.

19. Rakhmilevich AL, Timmins JG, Janssen K, Pohlmann EL, Sheehy MJ, Yang NS. Gene gunmediated IL-12 gene therapy induces antitumor effects in the absence of toxicity: a direct comparison with systemic IL-12 protein therapy. J Immunother. 1999 Mar;22(2):135-44.

20. Dow SW, Elmslie RE, Fradkin LG, Liggitt DH, Heath TD, Willson AP, et al. Intravenous cytokine gene delivery by lipid-DNA complexes controls the growth of established lung metastases. Hum Gene Ther. 1999 Dec 10;10(18):2961-72.
21. Whitmore M, Li S, Huang L. LPD lipopolyplex initiates a potent cytokine response and inhibits tumor growth. Gene Ther. 1999 Nov;6(11):1867-75.

22. Blezinger P, Freimark BD, Matar M, Wilson E, Singhal A, Min W, et al. Intratracheal administration of interleukin 12 plasmid-cationic lipid complexes inhibits murine lung metastases. Hum Gene Ther. 1999 Mar 20;10(5):723-31.

23. Chen W, Shao CS, Shen JG, Pan Y, Zheng S. [Therapeutic effect of cationic liposome-mediated interleukin-12 gene delivery on murine melanoma in vivo]. Zhejiang Da Xue Xue Bao Yi Xue Ban. 2005 Jan;34(1):73-6.

24. Horinaga M, Harsch KM, Fukuyama R, Heston W, Larchian W. Intravesical interleukin-12 gene therapy in an orthotopic bladder cancer model. Urology. 2005 Aug;66(2):461-6.

25. Faneca H, Cabrita AS, Simoes S, Pedroso de Lima MC. Evaluation of the antitumoral effect mediated by IL-12 and HSV-tk genes when delivered by a novel lipid-based system. Biochim Biophys Acta. 2007 May;1768(5):1093-102.

26. Speroni L, Gasparri J, de los ABV, Chiaramoni NS, Smagur A, Szala S, et al. Antitumoral effect of IL12 gene transfected via liposomes into B16F0 cells. Acta Biochim Pol. 2009;56(2):249-53.

27. Harada N, Shimada M, Okano S, Suehiro T, Soejima $\mathrm{Y}$, Tomita $\mathrm{Y}$, et al. IL-12 gene therapy is an effective therapeutic strategy for hepatocellular carcinoma in immunosuppressed mice. J Immunol. 2004 Dec 1;173(11):6635-44.

28. Daud AI, DeConti RC, Andrews S, Urbas P, Riker AI, Sondak VK, et al. Phase I trial of interleukin-12 plasmid electroporation in patients with metastatic melanoma. J Clin Oncol. 2008 Dec 20;26(36):5896903.

29. Zhu S, Li S. Systemic IL-12 gene therapy for treating malignancy via intramuscular electroporation. Methods Mol Biol. 2008;423:32737.

30. Ma X, Trinchieri G. Regulation of interleukin- 12 production in antigen-presenting cells. Adv Immunol. 2001;79:55-92.

31. Colombo MP, Trinchieri G. Interleukin-12 in antitumor immunity and immunotherapy. Cytokine Growth Factor Rev. 2002 Apr;13(2):155-68.

32. Ling P, Gately MK, Gubler U, Stern AS, Lin P, Hollfelder K, et al. Human IL-12 p40 homodimer binds to the IL-12 receptor but does not mediate biologic activity. J Immunol. 1995 Jan 1;154(1):11627.

33. Mazzolini G, Prieto J, Melero I. Gene therapy of cancer with interleukin-12. Curr Pharm Des. 2003;9(24):1981-91.

34. Koch F, Stanzl U, Jennewein P, Janke K, Heufler C, Kampgen E, et al. High level IL-12 production by 
murine dendritic cells: upregulation via MHC class II and CD40 molecules and downregulation by IL-4 and IL-10. J Exp Med. 1996 Aug 1;184(2):741-6.

35. Skeen MJ, Miller MA, Shinnick TM, Ziegler HK. Regulation of murine macrophage IL-12 production. Activation of macrophages in vivo, restimulation in vitro, and modulation by other cytokines. J Immunol. 1996 Feb 1;156(3):1196-206.

36. Xu D, Gu P, Pan PY, Li Q, Sato AI, Chen SH. NK and $\mathrm{CD} 8+\mathrm{T}$ cell-mediated eradication of poorly immunogenic B16-F10 melanoma by the combined action of IL-12 gene therapy and 4-1BB costimulation. Int J Cancer. 2004 Apr 20;109(4):499-506.

37. Rodolfo M, Colombo MP. Interleukin-12 as an adjuvant for cancer immunotherapy. Methods. 1999 Sep;19(1):114-20.

38. Fallarino F, Uyttenhove C, Boon T, Gajewski TF. Endogenous IL-12 is necessary for rejection of P815 tumor variants in vivo. J Immunol. $1996 \mathrm{Feb}$ 1;156(3):1095-100.

39. Noguchi Y, Richards EC, Chen YT, Old LJ. Influence of interleukin 12 on p53 peptide vaccination against established Meth A sarcoma. Proc Natl Acad Sci U S A. 1995 Mar 14;92(6):221923.

40. Rao JB, Chamberlain RS, Bronte V, Carroll MW, Irvine KR, Moss B, et al. IL-12 is an effective adjuvant to recombinant vaccinia virus-based tumor vaccines: enhancement by simultaneous B7-1 expression. J Immunol. 1996 May 1;156(9):3357-65.

41. Albini A, Brigati C, Ventura A, Lorusso G, Pinter M, Morini M, et al. Angiostatin anti-angiogenesis requires IL-12: the innate immune system as a key target. J Transl Med. 2009;7:5.

42. Siddiqui F, Ehrhart EJ, Charles B, Chubb L, Li CY, Zhang $X$, et al. Anti-angiogenic effects of interleukin-12 delivered by a novel hyperthermia induced gene construct. Int J Hyperthermia. 2006 Nov;22(7):587-606.

43. Duda DG, Sunamura M, Lozonschi L, Kodama T, Egawa S, Matsumoto G, et al. Direct in vitro evidence and in vivo analysis of the antiangiogenesis effects of interleukin 12. Cancer Res. 2000 Feb 15;60(4):1111-6.

44. Voest EE, Kenyon BM, O'Reilly MS, Truitt G, D'Amato RJ, Folkman J. Inhibition of angiogenesis in vivo by interleukin 12. J Natl Cancer Inst. 1995 Apr 19;87(8):581-6.

45. Brunda MJ, Luistro L, Rumennik L, Wright RB, Dvorozniak M, Aglione A, et al. Antitumor activity of interleukin 12 in preclinical models. Cancer Chemother Pharmacol. 1996;38 Suppl:S16-21.

46. Davidoff AM, Kimbrough SA, Ng CY, Shochat SJ, Vanin EF. Neuroblastoma regression and immunity induced by transgenic expression of interleukin-12.
Journal of pediatric surgery. 1999 May;34(5):902-6; discussion 6-7.

47. Tahara H, Zitvogel L, Storkus WJ, Zeh HJ, 3rd, McKinney TG, Schreiber RD, et al. Effective eradication of established murine tumors with IL-12 gene therapy using a polycistronic retroviral vector. J Immunol. 1995 Jun 15;154(12):6466-74.

48. Fallarino F, Ashikari A, Boon T, Gajewski TF. Antigen-specific regression of established tumors induced by active immunization with irradiated IL12- but not B7-1-transfected tumor cells. International immunology. 1997 Sep;9(9):1259-69.

49. Barker SE, Grosse SM, Siapati EK, Kritz A, Kinnon $\mathrm{C}$, Thrasher AJ, et al. Immunotherapy for neuroblastoma using syngeneic fibroblasts transfected with IL-2 and IL-12. British journal of cancer. 2007 Jul 16;97(2):210-7.

50. Lode HN, Dreier T, Xiang R, Varki NM, Kang AS, Reisfeld RA. Gene therapy with a single chain interleukin 12 fusion protein induces $\mathrm{T}$ celldependent protective immunity in a syngeneic model of murine neuroblastoma. Proc Natl Acad Sci U S A. 1998 Mar 3;95(5):2475-80.

51. Lode HN, Xiang R, Duncan SR, Theofilopoulos AN, Gillies SD, Reisfeld RA. Tumor-targeted IL-2 amplifies $\mathrm{T}$ cell-mediated immune response induced by gene therapy with single-chain IL-12. Proc Natl Acad Sci U S A. 1999 Jul 20;96(15):8591-6.

52. Siapati KE, Barker S, Kinnon C, Michalski A, Anderson R, Brickell P, et al. Improved antitumour immunity in murine neuroblastoma using a combination of IL-2 and IL-12. British journal of cancer. 2003 May 19;88(10):1641-8.

53. Croce M, Meazza R, Orengo AM, Radic L, De Giovanni B, Gambini C, et al. Sequential immunogene therapy with interleukin-12- and interleukin-15-engineered neuroblastoma cells cures metastatic disease in syngeneic mice. Clin Cancer Res. 2005 Jan 15;11(2 Pt 1):735-42.

54. Kang WK, Park C, Yoon HL, Kim WS, Yoon SS, Lee $\mathrm{MH}$, et al. Interleukin 12 gene therapy of cancer by peritumoral injection of transduced autologous fibroblasts: outcome of a phase I study. Hum Gene Ther. 2001 Apr 10;12(6):671-84.

55. Miller G, Lahrs S, Dematteo RP. Overexpression of interleukin-12 enables dendritic cells to activate NK cells and confer systemic antitumor immunity. The FASEB journal : official publication of the Federation of American Societies for Experimental Biology. 2003 Apr;17(6):728-30.

56. Shimizu T, Berhanu A, Redlinger RE, Jr., Watkins S, Lotze MT, Barksdale EM, Jr. Interleukin-12 transduced dendritic cells induce regression of established murine neuroblastoma. Journal of pediatric surgery. $2001 \mathrm{Aug;36(8):1285-92.}$ 
57. Iinuma $\mathrm{H}$, Okinaga $\mathrm{K}$, Fukushima $\mathrm{R}$, Inaba $\mathrm{T}$, Iwasaki K, Okinaga A, et al. Superior protective and therapeutic effects of IL-12 and IL-18 genetransduced dendritic neuroblastoma fusion cells on liver metastasis of murine neuroblastoma. J Immunol. 2006 Mar 15;176(6):3461-9.

58. Koido S, Hara E, Homma S, Namiki Y, Ohkusa T, Gong $\mathrm{J}$, et al. Cancer vaccine by fusions of dendritic and cancer cells. Clinical \& developmental immunology. 2009;2009:657369.

59. Orange JS, Salazar-Mather TP, Opal SM, Spencer RL, Miller AH, McEwen BS, et al. Mechanism of interleukin 12-mediated toxicities during experimental viral infections: role of tumor necrosis factor and glucocorticoids. J Exp Med. 1995 Mar 1;181(3):901-14.

60. Leonard JP, Sherman ML, Fisher GL, Buchanan LJ, Larsen G, Atkins MB, et al. Effects of single-dose interleukin-12 exposure on interleukin-12-associated toxicity and interferon-gamma production. Blood. 1997 Oct 1;90(7):2541-8.

61. Atkins MB, Robertson MJ, Gordon M, Lotze MT, DeCoste M, DuBois JS, et al. Phase I evaluation of intravenous recombinant human interleukin 12 in patients with advanced malignancies. Clin Cancer Res. 1997 Mar;3(3):409-17.

62. Yockman JW, Maheshwari A, Han SO, Kim SW. Tumor regression by repeated intratumoral delivery of water soluble lipopolymers/p2CMVmIL-12 complexes. J Control Release. 2003 Feb 21;87(13):177-86.

63. Tan J, Newton CA, Djeu JY, Gutsch DE, Chang AE, Yang NS, et al. Injection of complementary DNA encoding interleukin-12 inhibits tumor establishment at a distant site in a murine renal carcinoma model. Cancer Res. 1996 Aug 1;56(15):3399-403.

64. Car BD, Eng VM, Lipman JM, Anderson TD. The toxicology of interleukin-12: a review. Toxicol Pathol. 1999 Jan-Feb;27(1):58-63.

65. Maheshwari A, Mahato RI, McGregor J, Han S, Samlowski WE, Park JS, et al. Soluble biodegradable polymer-based cytokine gene delivery for cancer treatment. Mol Ther. 2000 Aug;2(2):12130 .

66. Mahato RI, Lee M, Han S, Maheshwari A, Kim SW. Intratumoral delivery of p2CMVmIL-12 using water-soluble lipopolymers. Mol Ther. 2001 Aug;4(2):130-8.

67. Fewell JG, Matar M, Slobodkin G, Han SO, Rice J, Hovanes B, et al. Synthesis and application of a non- viral gene delivery system for immunogene therapy of cancer. J Control Release. 2005 Dec 5;109(13):288-98.

68. Fewell JG, Matar MM, Rice JS, Brunhoeber E, Slobodkin G, Pence C, et al. Treatment of disseminated ovarian cancer using nonviral interleukin-12 gene therapy delivered intraperitoneally. J Gene Med. 2009 Aug;11(8):71828.

69. Mesiano S, Ferrara N, Jaffe RB. Role of vascular endothelial growth factor in ovarian cancer: inhibition of ascites formation by immunoneutralization. Am J Pathol. 1998 Oct;153(4):1249-56.

70. Hu L, Hofmann J, Holash J, Yancopoulos GD, Sood AK, Jaffe RB. Vascular endothelial growth factor trap combined with paclitaxel strikingly inhibits tumor and ascites, prolonging survival in a human ovarian cancer model. Clin Cancer Res. 2005 Oct 1;11(19 Pt 1):6966-71.

71. Wang Y, Gao S, Ye WH, Yoon HS, Yang YY. Codelivery of drugs and DNA from cationic core-shell nanoparticles self-assembled from a biodegradable copolymer. Nat Mater. 2006 Oct;5(10):791-6.

72. Kim TH, Jin H, Kim HW, Cho MH, Cho CS. Mannosylated chitosan nanoparticle-based cytokine gene therapy suppressed cancer growth in BALB/c mice bearing CT-26 carcinoma cells. Mol Cancer Ther. 2006 Jul;5(7):1723-32.

73. Diez S, Navarro G, de ICT. In vivo targeted gene delivery by cationic nanoparticles for treatment of hepatocellular carcinoma. J Gene Med. 2009 Jan;11(1):38-45.

74. Diez S, Migueliz I, Tros de Ilarduya C. Targeted cationic poly(D,L-lactic-co-glycolic acid) nanoparticles for gene delivery to cultured cells. Cell Mol Biol Lett. 2009;14(2):347-62.

75. Rodrigo-Garzon M, Berraondo P, Ochoa L, Zulueta JJ, Gonzalez-Aseguinolaza G. Antitumoral efficacy of DNA nanoparticles in murine models of lung cancer and pulmonary metastasis. Cancer Gene Ther. 2010 Jan;17(1):20-7.

76. Ogris M. Nucleic acid based therapeutics for tumor therapy. Anticancer Agents Med Chem. 2006 Nov;6(6):563-70.

77. Ogris M, Wagner E. Tumor-targeted gene transfer with DNA polyplexes. Somat Cell Mol Genet. 2002 Nov;27(1-6):85-95. 